given the other experimental variables employed. By staining the bacteria with a fluorescent dye, the researchers were able to monitor the manipulation of the bacteria with a fluorescence microscope.

By controlling the current in each wire of the matrix, versatile manipulation of the bacteria could be achieved. For example, the researchers transported multiple groups of bacteria independently along different paths and oriented individual bacterium. The assembled magnetic nanostructures, which were exposed when the researchers lysed the cells and removed the cellular membranes and other debris, were viewed with scanning electron microscopy. Examples of the nanoassemblies include a long chain of spherical nanomagnets and a ring of magnetic nanoparticles, resembling a pearl necklace, formed by trapping two bacteria.

The researchers said, "Using an electric field for manipulation, [our] method... can be extended to assemble other types of biogenic nanoparticles, for example, silver or gold nanocrystals in bacteria, or cadmium sulfide nanocrystals in yeast." They said that biomineralization and micromanipulation can be combined to grow and assemble nanoparticles into customized structures.

STEVEN TROHALAKI

\section{Organic-Inorganic Hybrid Electrolyte Membrane Achieves Thermal Stability above $100^{\circ} \mathrm{C}$}

Proton conductors are attractive materials because of their application in fuel cells, proton sensors, and ion-exchange membranes. However, currently available materials suffer from a decrease in conductivity at temperatures above $80^{\circ} \mathrm{C}$ and are unstable above $100^{\circ} \mathrm{C}$. A group of researchers in Japan from the Himeji Institute of Technology, the New Glass Forum Osaka Research Laboratory, the Kobe University of Mercantile Marine, the National Institute of Advanced Industrial Science and Technology, and Kyoto University has developed an organic-inorganic hybrid ionic conductive material with chemical resistance and thermal stability above $100^{\circ} \mathrm{C}$.

As reported in the March issue of the Journal of the American Ceramic Society ( $\mathrm{p}$. 504), T. Yazawa of the Himeji Institute of Technology and co-workers fabricated a porous glass by phase separation and later modified its surface by attaching thiol groups. The modification was performed by the reaction of the glass with (3-mercaptopropyl)trimethoxysilane. The thiol groups were later oxidized in concentrated $\mathrm{HNO}_{3}$ to obtain sulfonic acid groups, which are effective donors of protons. The pore size and pore volume of the samples were measured by nitrogen absorption isotherms; the researchers also studied their surface acidity and conductivity.
The conversion of the thiol groups on the surface upon oxidation to sulfonic acid produced an increase in the surface acidity. The intrinsic acidity constant $\mathrm{p} K_{\mathrm{a}}$

\title{
Circular Photonic Crystals Allow Formation of an Isotropic Photonic Bandgap
}

One of the main advantages of photonic crystals is their ability to inhibit spontaneous emission of light. An isotropic photonic bandgap has been previously proposed to suppress spontaneous emission in all directions. To date, two vehicles used to obtain an isotropic bandgap are quasi-crystals and amorphous (i.e., disordered) photonic materials. In the May 15 issue of Optics Letters (p. 1084), N. Horiuchi of RIKEN, T. Nozokido of Tohoku University, and their colleagues have reported that circular photonic crystals (CPC) are yet another route to the formation of an isotropic photonic gap. Disordered materials are inherently difficult to design for specific bandgaps due to their random nature. The advantage of the CPC over disordered materials is that the CPC has a very structured design that allows a priori determination of the bandgap.

The CPC was fabricated by carefully arranging $30 \mathrm{~mm}$ alumina rods (with a refractive index of 3.1) in concentric circles, similar to microstructured optical fiber. The number of circles was varied between 10 and 108 . While no translational symmetry exists in such a structure, triangular and square-like lattices appeared within different regions of the structure. In order to minimize this, a random phase shift from 0 to $\pi / 3$ of the sixfold symmetry for each circle was employed, as shown in the Figure. In this way, the distance between rods within a concentric circle is constant. Despite the phase shift, the nearest-neighbor distance also remains unchanged.

The transmission of a millimeter wave into the CPC was measured by irradiating the CPC with a transverse magneticpolarized wave incident from a direction orthogonal to the longitudinal axis of the rods. The signal strength inside the CPC was measured by placing a probe antenna within it. Multiple measurements were made, and the resulting transmission spectra showed a bandgap between $9.1 \mathrm{GHz}$ and 14.0 GHz. Theoretical calculations were in close agreement with the experimental findings. Angle-resolved measurements showed no significant change in the bandgap, indicating an isotropic photonic bandgap. It was found that an exterior part of CPC with a large number of concentric circles (108) yielded a $1 \mathrm{GHz}$ shift upwards in the lower band edge, but the bandgap was otherwise unaffected.

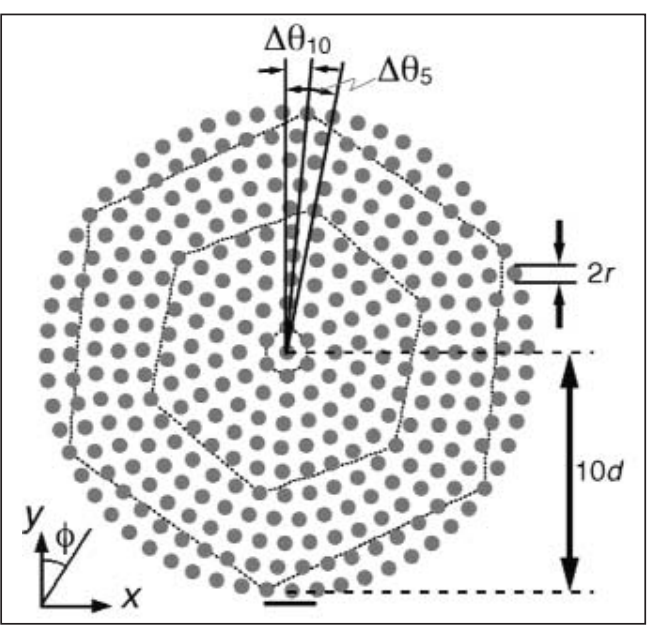

Figure. A circular photonic crystal composed of 10 concentric circles with a small phase shift $(\Delta \theta)$ to reduce the appearance of ordered regions. The bar at the bottom indicates the position of the probe antenna, $\mathrm{d}$ is the radial distance, and $\mathrm{r}$ is the radius of the rods. The angle of the incidence of the millimeter wave $\phi$ is zero when parallel to the $y$ axis.

The researchers said that the

isotropic bandgap is found not only at the axis of the concentric circles, but throughout the structure. This suggests, they said, that the isotropic photonic bandgap is the result of the short-range order of the CPC. The research team said that a practical application of this technology, beyond that of suppressing spontaneous emission, is the ability to create two-dimensional waveguides by line defects with arbitrary bend, since the technology is not restricted to the photonic crystal lattice.

JEFFREY DiMAIO 